\title{
Descripción del consumo de nutrientes según el nivel de procesamiento industrial de los alimentos. Encuesta Nacional de Nutrición y Salud - 2005 Description of nutrient consumption based on the level of industrial food processing. National Survey on Nutrition and Health of 2005
}

\author{
Lic. Ignacio Drakea , Dr. Enrique Abeyá Gilardon ${ }^{a}$, Mag. Guadalupe Mangialavoria \\ y Lic. Ana Biglieri ${ }^{a}$
}

\begin{abstract}
RESUMEN
Los avances tecnológicos y el cambio de estilos de vida se reflejan en mayor consumo de alimentos procesados y ultraprocesados respecto de los naturales. Con datos de la Encuesta Nacional de Nutrición y Salud, se describe el consumo de esos alimentos y su relación con condiciones de vida en tres grupos etarios de Argentina (niñas y niños de 6-23 meses, niñas y niños de 2-5 años y mujeres de 10-49 años).

El consumo en niños de 6-23 meses es alto en nutrientes provenientes de alimentos naturales: calcio $(75,9 \%)$, proteínas $(72,7 \%)$, hierro $(71,5 \%)$ y fibra $(68,9 \%)$. En niños de $2-5$ años, desciende significativamente. En las mujeres, disminuye aún más. La proporción del aporte de energía de productos ultraprocesados es mayor en la población de más altos ingresos respecto de los bajos ingresos en todos los grupos poblacionales. Palabras clave: industria dealimentos, epidemiología, Argentina, alimentos, encuestas nutricionales.
\end{abstract}

http: / / dx.doi.org/10.5546/ aap.2018.345

Texto completo en inglés:

http: / / dx.doi.org/10.5546/ aap.2018.eng.345

Cómo citar: Drake I, Abeyá Gilardon E, Mangialavori G, Biglieri A. Descripción del consumo de nutrientes según el nivel de procesamiento industrial de los alimentos. Encuesta Nacional de Nutrición y Salud - 2005. Arch Argent Pediatr

Área de Nutrición Dirección Nacional de Maternidad,

Infancia y

Adolescencia, Ciudad de Buenos Aires, Argentina.

Correspondencia: Lic. Ignacio Drake: ignacio.drake@gmail. com

Financiamiento: Ninguno.

Conflicto de intereses: Ninguno que declarar.

Recibido: 28-7-2017

Aceptado: 14-2-2018 morbilidad no afecta a todos por igual, sino de manera inequitativa: son más afectadas las poblaciones con bajos recursos socioeconómicos. ${ }^{4-6}$

Desde hace tiempo, la industria de la alimentación ha favorecido la proliferación de productos ultraprocesados con desarrollos tecnológicos que refuerzan los aspectos positivos de sabor, textura, aroma, etc., pero que son poco saludables y tienen consecuencias en las ECNT. ${ }^{7,8}$ Asimismo, los productos ultraprocesados contienen menos proteínas, fibras, más azúcares libres, sodio, grasas totales y saturadas y alta densidad energética, cuyo consumo elevado promueve perfiles de nutrición muy perjudiciales. La propuesta de categorizar los alimentos según su nivel de procesamiento ganó consenso e importancia para diferenciar los patrones alimentarios de las poblaciones. ${ }^{8-10}$

En las últimas dos décadas, sobre la base de las Encuestas de Gasto de los Hogares del Instituto Nacional de Estadística y Censos (INDEC), se observa un claro desplazamiento de la alimentación tradicional basada en alimentos frescos o mínimamente procesados hacia un patrón alimentario basado en alimentos ultraprocesados. El cambio conlleva un deterioro en la calidad de la ingesta con modificaciones en los consumos de fibra, grasas, sodio y azúcares libres de significativa importancia ${ }^{11} \mathrm{y}$ una reducción importante en el aporte de vitaminas y minerales. 
La Encuesta Nacional de Nutrición y Salud (ENNyS) de Argentina realizada en 2005 aportó un panorama de la situación nutricional de distintos grupos poblacionales, pero no se analizó desde la perspectiva del procesamiento tecnológico de los alimentos. El objetivo del presente artículo fue describir la ingesta alimentaria de grupos etarios seleccionados de la población argentina utilizando la clasificación NOVA, ${ }^{12}$ que tomaba en cuenta el nivel de procesamiento industrial de los alimentos y lo relacionaba con variables socioeconómicas y geográficas.

\section{POBLACIÓN Y MÉTODOS}

La ENNyS se realizó mediante un muestreo probabilístico multietápico de grupos etarios independientes en localidades de más de 5000 habitantes de todo el país. El detalle del diseño muestral y los grupos poblacionales de la ENNyS, así como los aspectos metodológicos, fueron explicados en publicaciones previas. ${ }^{13,14} \mathrm{El}$ estudio que aquí se presenta se realizó sobre los registros de ingesta alimentaria. La ingesta fue estimada mediante el método del recordatorio de las últimas 24 horas $(\mathrm{R} 24 \mathrm{H})$ para niños y niñas de 6 a 23 meses, de 2 a 5 años y mujeres de 10 a 49 años representativas de la población para cada una de las seis regiones de Argentina.

Se utilizaron los indicadores de condiciones de vida siguiendo la metodología del INDEC para la estimación de las necesidades básicas insatisfechas (NBI), ${ }^{15}$ línea de indigencia y de pobreza $(\mathrm{LI} / \mathrm{LP})^{16}$ y la distribución quintílica del ingreso per cápita familiar.

Los alimentos fueron categorizados según la clasificación NOVA de alimentos. Esta reúne los alimentos en cuatro grupos de acuerdo con la naturaleza, extensión y propósito de los procesos de su producción y/o conservación, respondiendo a las siguientes características:

- Grupo 1. Alimentos no procesados o mínimamente procesados: se definen como partes de plantas o animales que no han sido procesados industrialmente o han sido modificados de manera que no agregan ninguna sustancia nueva (como grasas, azúcar o sal), pero pueden implicar la eliminación de partes del alimento. Por ejemplo, frutas o verduras, carne fresca o congelada, huevos, leche y arroz $\mathrm{u}$ otros granos.

- Grupo 2. Ingredientes culinarios procesados: son sustancias extraídas de alimentos no procesados, como aceite y azúcar, u obtenidos de la naturaleza, como la sal. Los ingredientes culinarios normalmente no se consumen solos, pero se usan en combinación con alimentos no procesados y mínimamente procesados en la cocina para preparar platos y comidas.

- Grupo 3. Alimentos procesados: se producen agregando sal, aceite, azúcar u otros ingredientes culinarios a los alimentos mínimamente procesados. Los alimentos procesados siguen siendo reconocibles como versiones modificadas de alimentos no procesados e incluyen elementos tales como frutas o vegetales enlatados, nueces saladas, carnes curadas o ahumadas y queso.

- Grupo 4. Alimentos ultraprocesados: se definen como formulaciones industriales de ingredientes múltiples e incluyen bebidas azucaradas, panes envasados, galletas, aperitivos salados, golosinas, helados, cereales para el desayuno y comidas congeladas listas para consumir.

Cada alimento mencionado en el $\mathrm{R} 24 \mathrm{H}$ fue asignado de manera independiente por cada una de las nutricionistas del equipo a uno de los cuatro grupos anteriores. Las discrepancias se resolvieron por consenso.

Asimismo, la composición química de cada alimento es la consignada en la base de datos de composición de alimentos del sistema de análisis y registro de alimentos (SARA). Está integrada por 379 alimentos. Para estos, la base cuenta con información de energía, proteínas, lípidos, hidratos de carbono disponibles, fibra, sodio, calcio, hierro, ácidos grasos (AG) saturados, AG monoinsaturados, AG poliinsaturados, entre otros.

Para el cálculo de porcentajes, en el numerador, se utilizó la suma de energía y nutrientes aportados por cada uno de los alimentos ingeridos según cada categoría de la clasificación NOVA y, para el denominador, se realizó la suma de energía y / o nutrientes aportados por todos los alimentos registrados en el R24H.

\section{RESULTADOS}

El número de casos encuestados según región y grupo etario se presenta en la Tabla 1 del Anexo (véase en formato electrónico).

\section{Grupo etario: de 6 a 23 meses}

En las comidas de los niños más pequeños, el mayor aporte de energía y nutrientes proviene de alimentos naturales e ingredientes (Figura 1). En este sentido, el 75,9\% del calcio, el 72,7\% de las proteínas, el 71,5\% del hierro y el $68,9 \%$ de la fibra 
están provistos por el grupo 1 de la clasificación NOVA. Asimismo, es baja la proporción de los productos procesados, salvo en el caso de los AG saturados $(63,4 \%)$, de los hidratos de carbono $(43,9 \%)$ y del sodio $(40,3 \%)$.

Si solo se considera el grupo 4 -ultraprocesados-, las diferencias de ingesta de energía y nutrientes según las NBI son significativas y varían hasta un máximo de 10 puntos porcentuales entre los niños que tienen NBI y los que no; algo similar sucede si se analiza por LP/LI. En tanto que, en los quintiles de ingreso más altos, aumenta el aporte de energía proveniente de productos ultraprocesados: 19,5\% en el quintil 1 de más bajos ingresos versus 32,9\% en el quintil 5 de más altos ingresos (Figura 2). Si bien la proporción es menor que lo que se observa en otros grupos poblacionales, se verifica que la capacidad de generar ingresos afecta los tipos de consumo de productos procesados, aún entre la población de menos edad.

Considerando el consumo de estos niños según su distribución geográfica, se observa que los menores aportes de energía provenientes de productos ultraprocesados se registran en el Noroeste Argentino (NOA) y Noreste Argentino (NEA) (alrededor del 19\%), seguidos por Cuyo y la región Pampeana (25\%), y los mayores, en el Área Metropolitana de Buenos Aires (AMBA) y la Patagonia (31\%).
El promedio del contenido nutricional de los alimentos consumidos para este grupo etario según el grupo NOVA se presenta en la Tabla 2 del Anexo (véase en formato electrónico).

\section{Grupo etario: de 2 a 5 años}

Los niños de esta edad, a diferencia del anterior, ya incorporaron plenamente alimentos sólidos del resto del grupo familiar. Se observa que la ingesta de energía y nutrientes de alimentos naturales e ingredientes disminuye significativamente; el 72,3\% del calcio, el 67,2\% de las proteínas, el 60,4\% del hierro y el 56,5\% de la fibra provienen de este grupo de alimentos (Figura 3). En tanto que la ingesta de nutrientes provenientes de productos ultraprocesados se mantuvo en los mismos niveles que en el grupo anterior.

Se observa un marcado aumento en el consumo de productos ultraprocesados entre los niños de esta edad que viven en hogares con mejores condiciones de vida, tanto en los que no tienen NBI como en los no pobres. En ambos casos, aumenta significativamente la brecha entre los que más privaciones tienen y los que menos tienen, incluso mayor que en el grupo anterior. En este mismo sentido, se observa una baja importante en la ingesta de energía proveniente de los alimentos naturales (grupo 1) de los quintiles de más altos ingresos -alrededor de 10 puntos porcentuales-

FIGURA 1. Distribución relativa de energía y nutrientes según la clasificación NOVA en niños y niñas de 6 a 23 meses (en porcentajes). $N=6035$

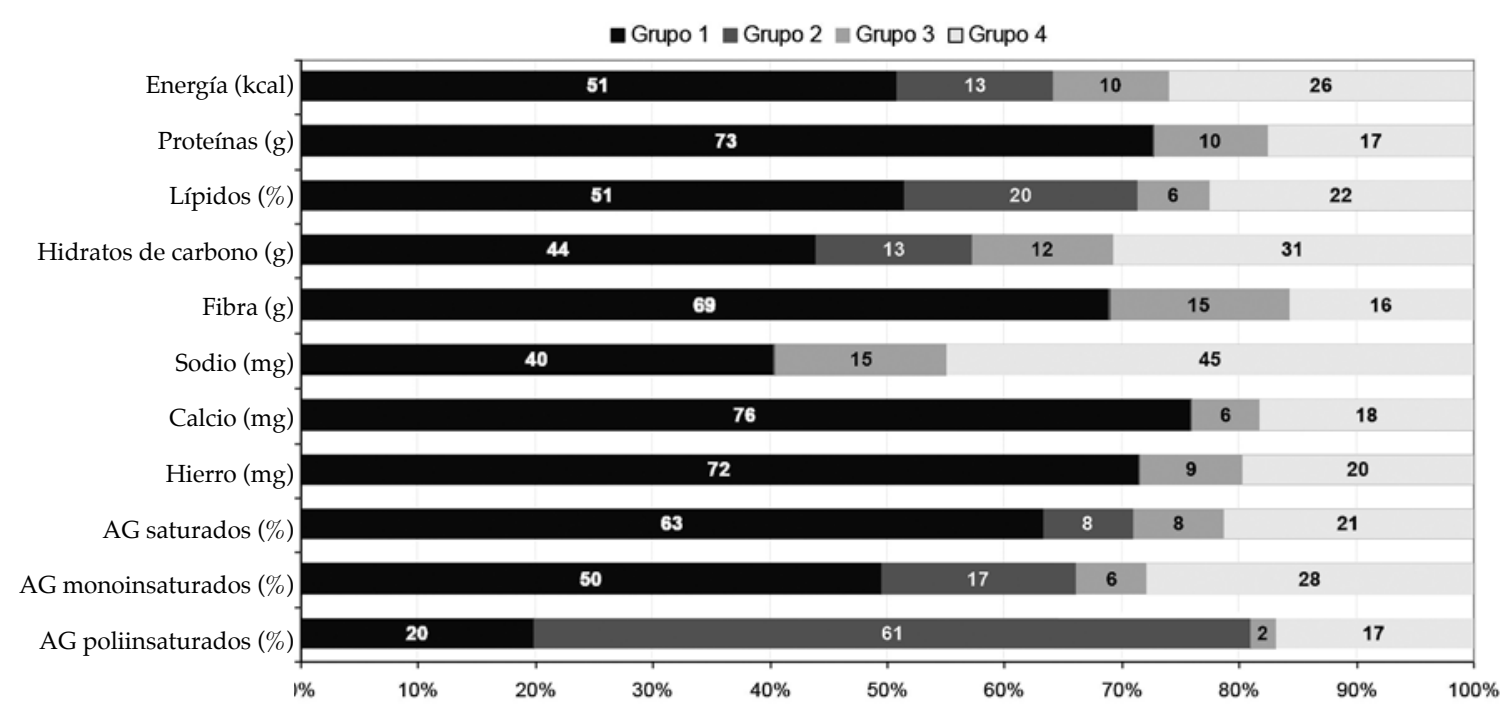

Fuente: Elaboración propia con datos de ENNyS (2005) y Monteiro et al. (2011).

AG: ácidos grasos. 
y un salto en la provisión de energía gracias al mayor consumo de productos procesados y ultraprocesados (grupo 3 y 4 ) entre los quintiles de más altos ingresos (Figura 4).

En la incorporación de energía por parte de los niños, en todas las regiones, se observó que el aporte proporcional de energía fue mayor a partir de alimentos ultraprocesados a expensas de los naturales. Al igual que entre los niños de 6 a 23 meses, el mayor consumo de productos

FIGURA 2. Distribución relativa de energía por quintiles de ingreso per cápita familiar según la clasificación NOVA en niños y niñas de 6 a 23 meses (en porcentajes). N=6035

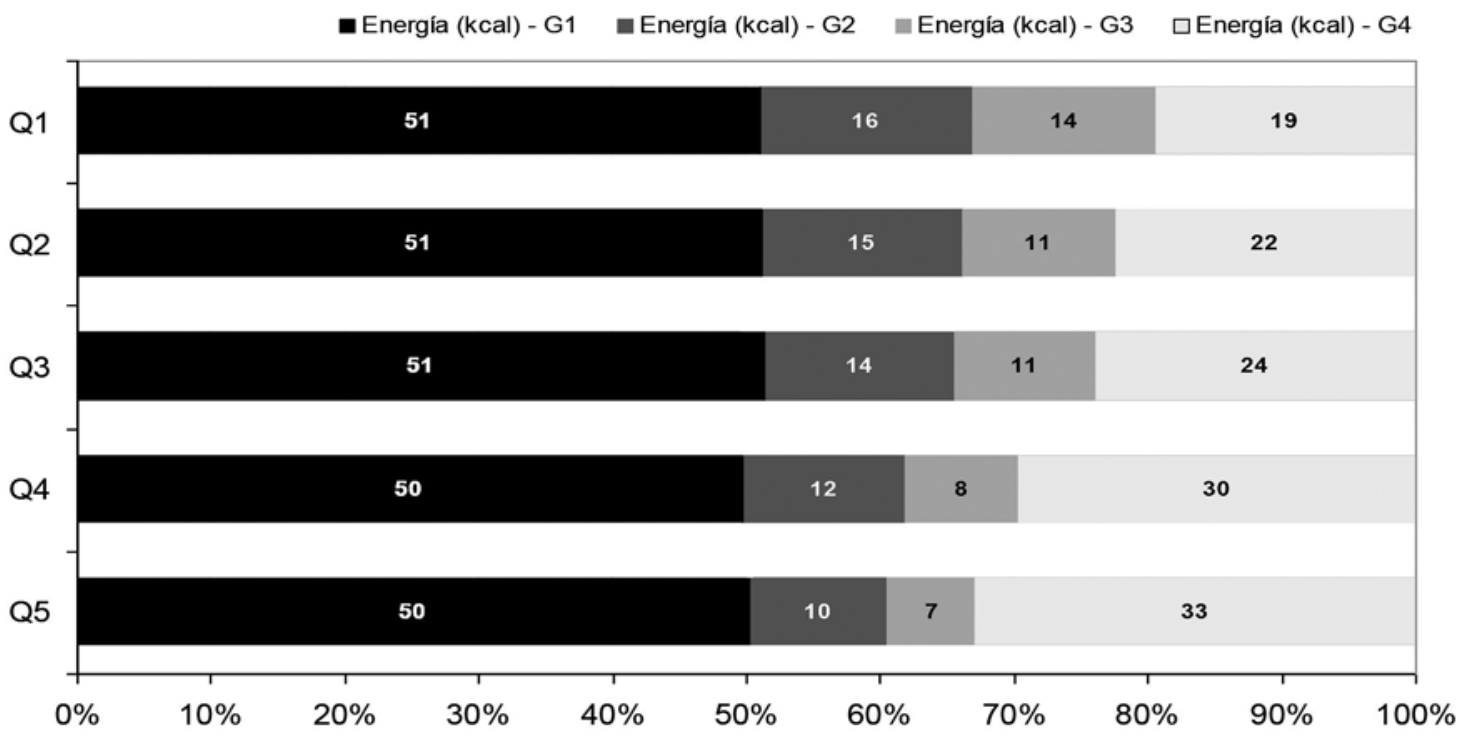

Fuente: Elaboración propia con datos de ENNyS (2005) y Monteiro et al. (2011).

Q: quintil; G: grupo; Kcal: kilocaloría.

FIGURA 3. Distribución relativa de energía y nutrientes según la clasificación NOVA en niños y niñas de 2 a 5 años (en porcentajes). $N=7364$

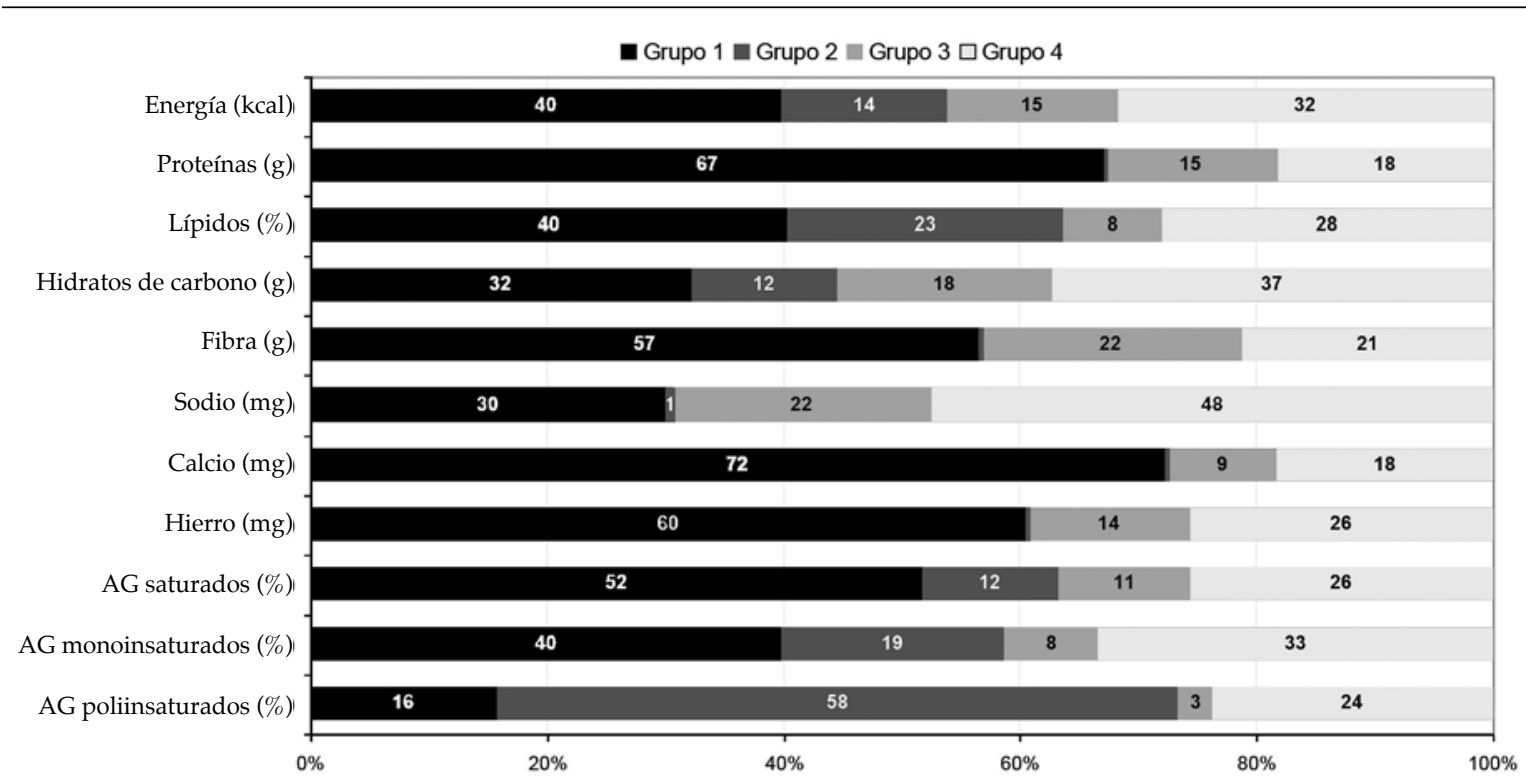

Fuente: Elaboración propia con datos de ENNyS (2005) y Monteiro et al. (2011).

AG: ácidos grasos. 
procesados y ultraprocesados se registra entre los niños que viven en el AMBA y la Patagonia. En este tipo de productos, los niños de estas regiones tienen los valores de ingesta más altos del país.

El promedio del contenido nutricional de los alimentos consumidos para este grupo etario según el grupo NOVA se presenta en la Tabla 3 del Anexo (véase en formato electrónico).

\section{Grupo etario: mujeres de 10 a 49 años}

Al realizar el análisis de este grupo, existe también la posibilidad de tomarlo como referencia para el consumo de alimentos de la población adulta. Se observa que la ingesta de energía y nutrientes a través de alimentos naturales (grupo 1) es aún menor que la del grupo de niños y niñas de 2 a 5 años. En este sentido, solo el 59,0\% de las proteínas, el 55,1\% del hierro, el $51,6 \%$ de fibra y el $46,4 \%$ del calcio provienen de alimentos naturales (Figura 5). Este grupo de mayor edad es el que menos aportes de nutrientes registra en este tipo de alimentos entre todos los grupos analizados.

En relación con la ingesta de nutrientes de productos ultraprocesados, se observa que las mujeres con menos privaciones socioeconómicas -sin NBI o no pobres- adquieren mayor proporción de nutrientes provenientes de este tipo de productos. Este aporte es muy similar al que tienen estos productos entre los niños y niñas de 2 a 5 años. En cuanto al consumo de mujeres diferenciado por quintiles de ingreso, se observa que, en todos los estratos, el aporte de energía a partir de los alimentos naturales o mínimamente procesados es menor que en el grupo anterior -alrededor de 10 puntos porcentuales menos(Figura 6). En tanto que el aporte de energía proveniente de productos ultraprocesados es significativamente mayor a medida que aumenta el nivel de ingresos.

En cuanto a las diferencias de consumo según la ubicación geográfica, las mujeres de prácticamente todas las regiones incorporan alrededor de un $30 \%$ de energía a través de alimentos poco procesados o naturales; la única excepción es el NEA, con un aporte mayor del $35,9 \%$. Esto se acompaña con una menor ingesta de energía a través de productos ultraprocesados en las regiones NOA, NEA y Cuyo (alrededor del $26 \%$ ) y un aporte de energía significativamente mayor a partir de este tipo de productos en la Patagonia y el AMBA (alrededor del 36\%).

El promedio del contenido nutricional de los alimentos consumidos para este grupo etario

FIGURA 4. Distribución relativa de energía por quintiles de ingreso per cápita familiar según la clasificación NOVA en niños y niñas de 2 a 5 años (en porcentajes). $N=7364$

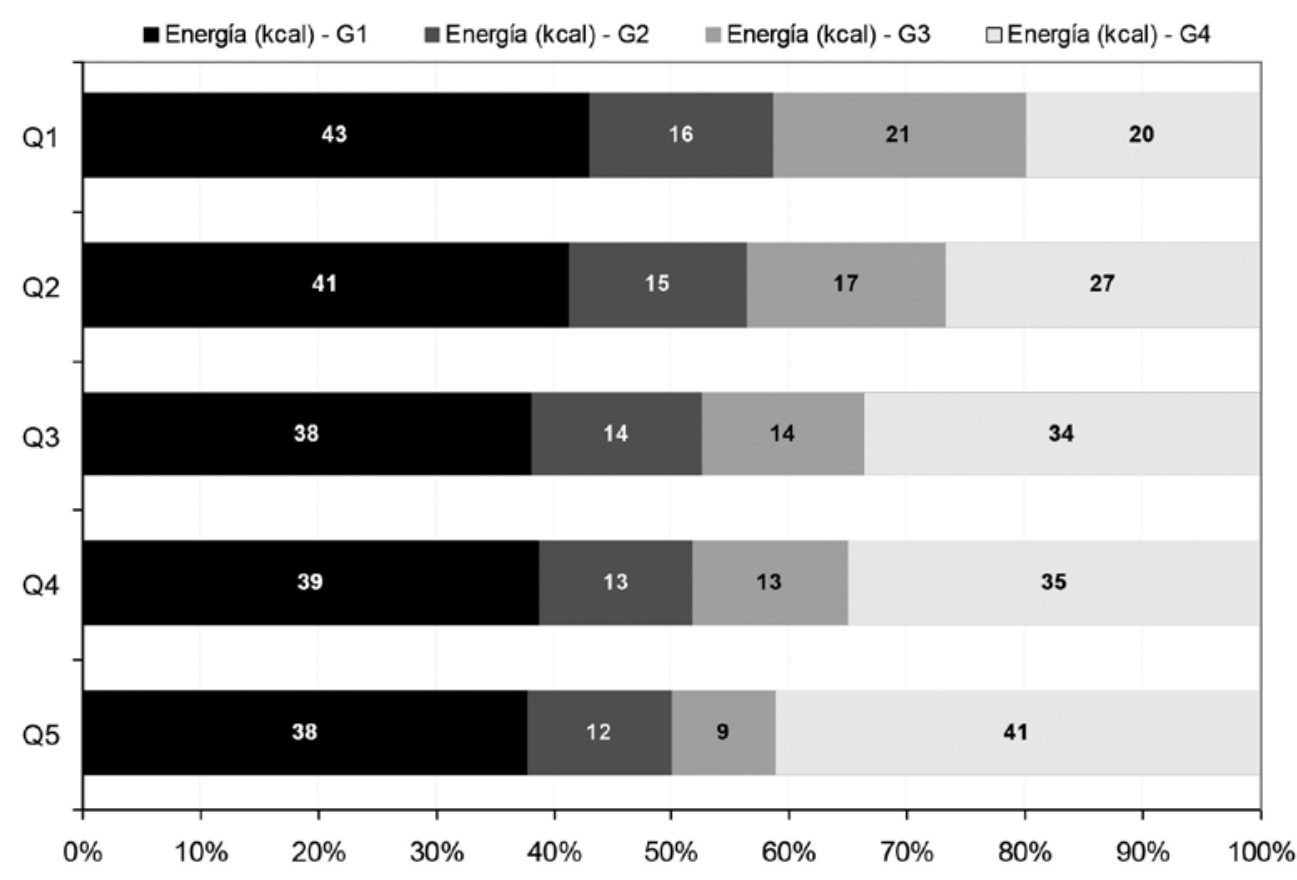

Fuente: Elaboración propia con datos de ENNyS (2005) y Monteiro et al. (2011).

Q: quintil; G: grupo; Kcal: kilocaloría. 
según el grupo NOVA se presenta en la Tabla 4 del Anexo (véase en formato electrónico).

\section{DISCUSIÓN}

Es amplia y variada la bibliografía sobre la descripción de la ingesta alimentaria según la composición química de los alimentos que ha permitido identificar y dimensionar los requerimientos de nutrientes y la magnitud de sus deficiencias. Esta descripción de la ingesta según la composición química es la que orientó el análisis de los resultados de la ENNyS en 2007. Existen similares marcos de referencia que clasifican los alimentos según su nivel

FIGURA 5. Distribución relativa de energía y nutrientes según la clasificación NOVA en mujeres de 10 a 49 años (en porcentajes). $N=6605$

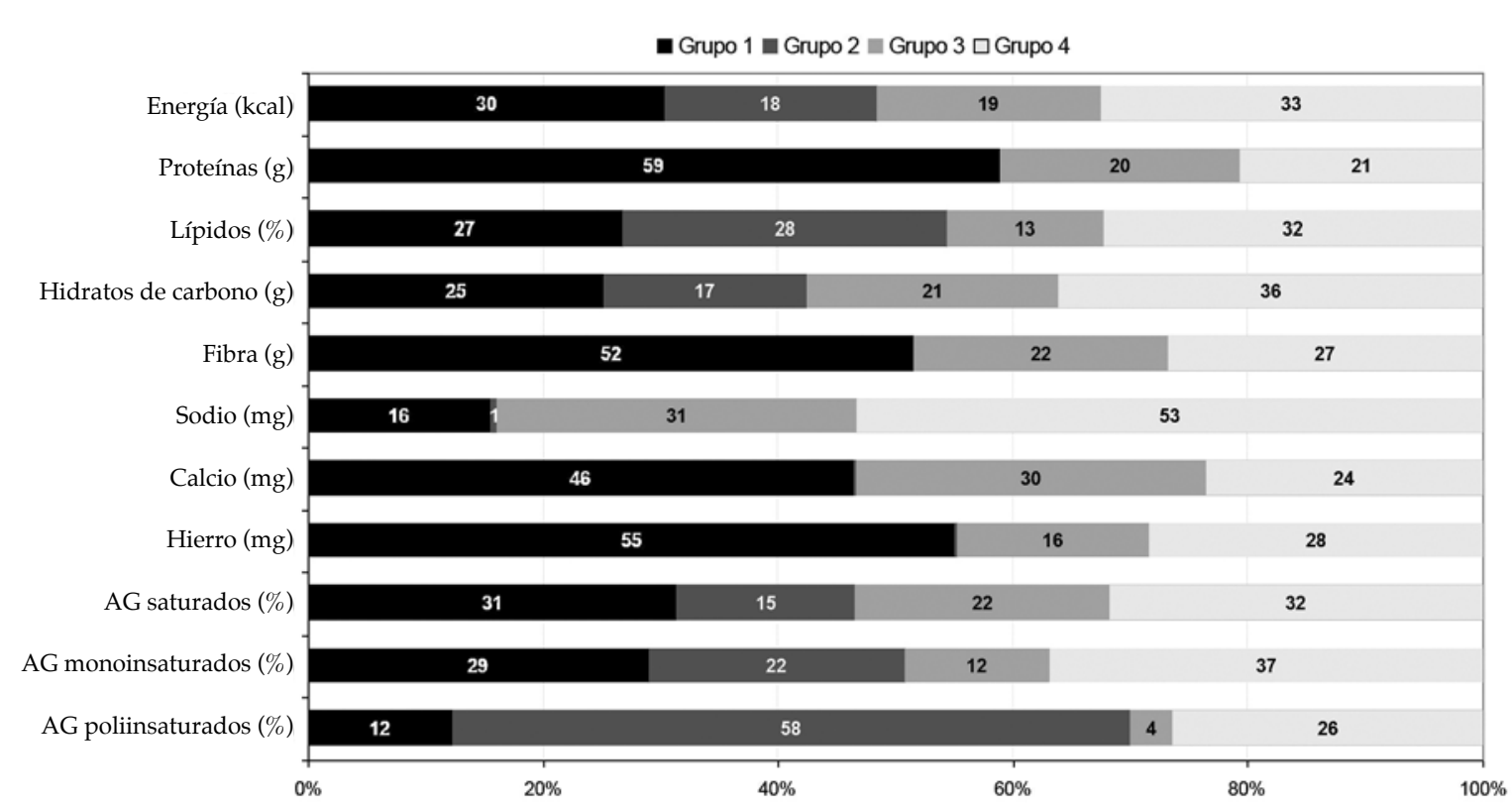

Fuente: Elaboración propia con datos de ENNyS (2005) y Monteiro et al. (2011).

AG: ácidos grasos.

FIGURA 6. Distribución de energía por quintiles de ingreso per cápita familiar según la clasificación NOVA en mujeres de 10 a 49 años. $N=6605$

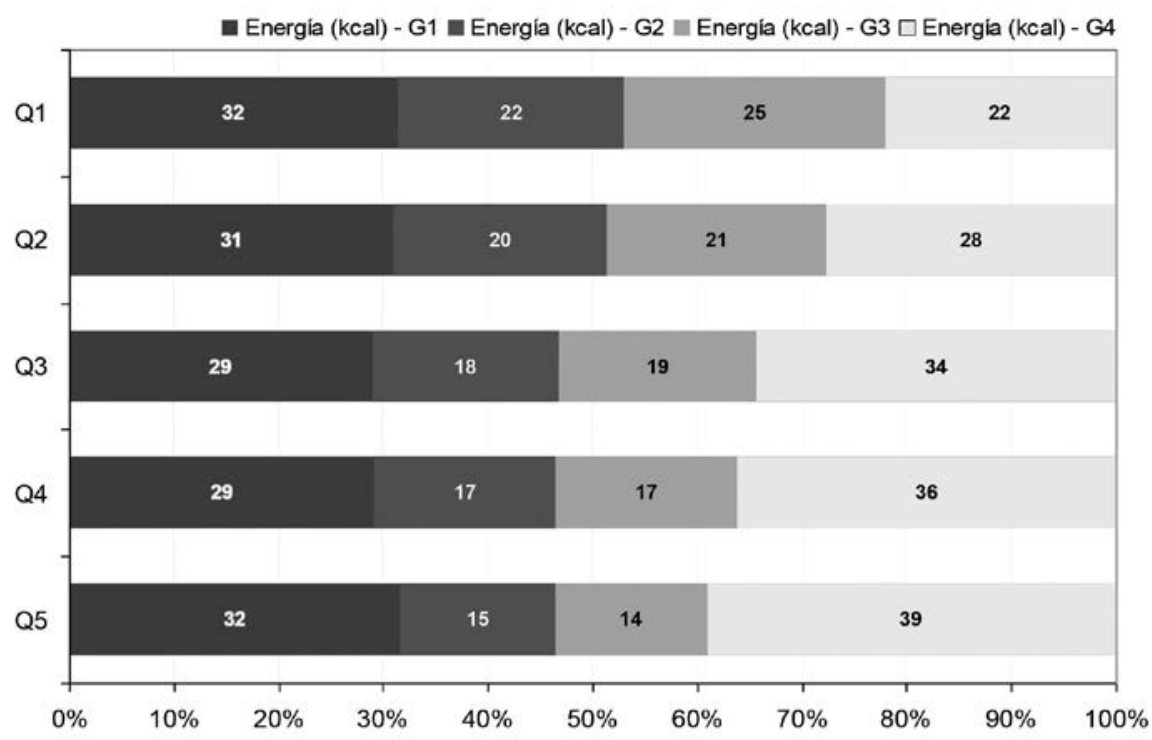

Fuente: Elaboración propia con datos de ENNyS (2005) y Monteiro et al. (2011).

Q: quintil; G: grupo; Kcal: kilocaloría. 
de procesamiento. ${ }^{17,18} \mathrm{El}$ más apropiado nos parece el sistema NOVA, que desde 2014 ha aumentado considerablemente en PubMed de Medline las publicaciones que utilizan la descripción de la ingesta desde este marco de referencia. Este sistema de clasificación de alimentos según su nivel de procesamiento ha ido ganando reconocimiento en la bibliografía hasta la publicación de un número especial en enero de 2018 de la revista líder en políticas públicas de nutrición ${ }^{19}$ y como parte de informes de la Organización Panamericana de la Salud (OPS) y la Food and Agriculture Organization (FAO), ${ }^{9,20}$

Hay suficiente evidencia epidemiológica que demuestra que el consumo de alimentos ultraprocesados se asocia con baja calidad de la dieta y malas condiciones de salud..$^{21} \mathrm{El}$ análisis del aporte de energía y nutrientes según el nivel de procesamiento aporta datos útiles para comprender e implementar políticas sanitarias que tiendan a mejorar la situación nutricional de la población de un país. ${ }^{22} \mathrm{Si}$ bien no hay datos actuales de consumos en Argentina, el análisis de la ingesta a través de los datos disponibles en la ENNyS permite conocer cómo es el consumo en edades críticas, como son los niños menores de 6 años y las mujeres en edad fértil.

La información aquí analizada de ingesta alimentaria, si bien se refiere a 12 años atrás, es la única disponible en Argentina con representatividad probabilística y sirve como línea de base para la comparación con próximas encuestas para observar cambios a través del tiempo. A partir de los grupos poblacionales analizados, se observa que, a medida que se incrementa la edad de la población, también aumenta la contribución de energía y ciertos nutrientes a partir de alimentos ultraprocesados.

La contribución de los alimentos ultraprocesados a la ingesta energética del presente estudio fue superior a la estimada para Brasil con datos de encuesta de gasto en los hogares para 2008-2009 equivalente a 21,5\% del consumo energético cuando, para nuestras estimaciones, es de $26 \%$ y $32 \%$ en niños de 6 a 23 meses y niños de 2 a 5 años, respectivamente, y de $33 \%$ en mujeres de 10 a 49 años. ${ }^{23}$ Además, en un estudio ecológico transversal de 19 países europeos, la mediana de contribución del consumo de energía por ultraprocesados en los hogares fue de $26,4 \% .^{24}$

Existe en este estudio una fuerte relación directa entre la contribución de ultraprocesados a la ingesta energética y el nivel del ingreso familiar, es decir, a mayor ingreso familiar per cápita, es mayor la proporción de la ingesta energética proveniente de los alimentos ultraprocesados. Esta relación directa entre ingreso familiar y consumo de ultraprocesados ha sido observada también en Brasil. Además, esta relación directa se da entre países, ya que, a mayor ingreso nacional, mayor proporción de consumo de ultraprocesados. ${ }^{25}$

De los nutrientes analizados es el sodio el que en mayor proporción es aportado por los alimentos ultraprocesados. En el caso de los AG poliinsaturados, se incluyen las grasas trans, que la industria de alimentos de Argentina, en 2005, aún las utilizaba como aceite vegetal hidrogenado por ser el más económico para elaborar alimentos procesados y ultraprocesados. Es por esto por lo que la contribución de los no procesados al consumo de estos AG aparece como más importante. En el año 2010, ya se incorporó su prohibición al Código Alimentario Argentino. ${ }^{26}$ La intervención regulatoria desde el Estado ha logrado descender los consumos de AG trans desde entonces.

La fortaleza de este estudio es la estimación de los consumos en una muestra probabilística de grupos poblacionales que son prioritarios para las políticas de nutrición pública desde el marco de referencia del nivel de procesamiento y de sobrepeso y obesidad en la población argentina. ${ }^{27-30}$ El análisis de las dos últimas décadas a través de las encuestas de gasto de los hogares de Argentina muestra un progresivo avance hacia los alimentos procesados desde 19\% de la ingesta calórica en 1996-1997 a $29 \%$ en 2012-2013. ${ }^{31}$

$\mathrm{Su}$ debilidad es que los consumos fueron registros únicos por individuo, sin la estimación de la variabilidad intraindividual, lo cual produce cierta incertidumbre en las estimaciones de los intervalos de confianza de las estimaciones de las medias. ${ }^{32}$

El monitoreo de la contribución de los ultraprocesados a la ingesta energética habitual puede ser una estrategia útil para orientar la promoción de políticas públicas por hábitos alimentarios más saludables. Se sabe que el consumo de ultraprocesados se asocia con mala calidad de la dieta y este ha sido el argumento fundamental para proponerlo como indicador poblacional de calidad de la dieta. ${ }^{33,34}$

\section{CONCLUSIÓN}

El aporte de energía y nutrientes provenientes de alimentos naturales o mínimamente procesados es mayoritario en los primeros años de vida. A medida que se consideran grupos poblacionales de mayor edad, la incorporación de ultraprocesados aumenta. El consumo de este 
tipo de productos es más frecuente dentro de los niveles socioeconómicos más altos o con mejores condiciones de vida.

\section{Agradecimientos}

A la Lic. Luciana Paduano por su colaboración en la asignación de alimentos a la clasificación NOVA y al Lic. Carlos Defrancisco por el procesamiento y armado de la base de datos.

\section{REFERENCIAS}

1. Ng M, Fleming T, Robinson M, et al. Global, regional, and national prevalence of overweight and obesity in children and adults during 1980-2013: a systematic analysis for the Global Burden of Disease Study 2013. Lancet 2014; 384(9945):766-81.

2. NCD RiskFactorCollaboration(NCD-RisC). Trendsinadult body-mass index in 200 countries from 1975 to 2014: a pooled analysis of 1698 population-based measurement studies with $19 \cdot 2$ million participants. Lancet 2016; 387(10026):1377-96.

3. Vandevijvere S, Chow CC, Hall KD, et al. Increased food energy supply as a major driver of the obesity epidemic: a global analysis. Bull World Health Organ 2015; 93(7):446-56.

4. Wang Y, Beydoun MA. The obesity epidemic in the United States- gender, age, socioeconomic, racial/ethnic, and geographic characteristics: a systematic review and metaregression analysis. Epidemiol Rev 2007;29:6-28.

5. Wang Y, Lobstein T. Worldwide trends in childhood overweight and obesity. Int J Pediatr Obes 2006;1(1):11-25.

6. Wang Y. Disparities in pediatric obesity in the United States. Adv Nutr 2011; 2(1):23-31.

7. Breslin PA. An evolutionary perspective on food and human taste. Curr Biol 2013; 23(9):R409-18.

8. FAO/OPS. América Latina y el Caribe: Panorama de la seguridad alimentaria y nutricional. Sistemas alimentarios sostenibles para poner fin al hambre y la malnutrición. Santiago: OPS; 2017. [Acceso: 15 de febrero de 2018]. Disponible en: http: / / www.fao.org/3/a-i6747s.pdf.

9. OPS/OMS. Alimentosy bebidasultraprocesadosen América Latina: tendencias, efecto sobre la obesidad e implicaciones para las políticas públicas. Washington: OPS; 2015. [Acceso: 15 de febrero de 2018]. Disponible en: iris.paho.org/xmlui/ bitstream/handle/123456789/7698/9789275318645_esp.pdf.

10. Ludwig DS. Technology, diet, and the burden of chronic disease. JAMA 2011; 305(13):1352-3.

11. Zapata ME, Rovirosa A, Carmuega E. La mesa Argentina en las últimas dos décadas: cambios en el patrón de consumo de alimentos y nutrientes 1996-2013. Ciudad Autónoma de Buenos Aires: Centro de Estudios sobre Nutrición Infantil - CESNI; 2016.

12. Monteiro CA, Cannon G, Levy R, et al. Food classification. Public Health. NOVA. The star shines bright. World Nutr. 2016;7(1-3):28-38. [Acceso: 18 de diciembre de 2017]. Disponible en: https: / / worldnutritionjournal.org/index. $\mathrm{php} / \mathrm{wn} /$ article/view/5/4.

13. Durán P, Mangialavori G, Biglieri A, et al. Estudio descriptivo de la situación nutricional en niños de 6-72 meses de la República Argentina. Resultados dela Encuesta Nacional de Nutrición y Salud (ENNyS). Arch Argent Pediatr 2009; 107(5): 397-404.

14. Ministerio de Salud. Encuesta Nacional de Nutrición y Salud. Documento de Resultados 2007. Buenos Aires: Ministerio deSalud, 2007. [Acceso: 18 de diciembre de 2017]. Disponible en: http: / / datos.dinami.gov.ar/produccion / nutricion/ennys.html.

15. INDEC. Mapa de necesidades básicas insatisfechas 2001. Aquí se Cuenta. 2003; 7. [Acceso: 15 de febrero de 2018]. Disponible en: https: / / www.indec.gov.ar/micro_sitios / webcenso/aquisecuenta/aqui7.pdf.
16. Dirección Nacional de Encuestas de Hogares del INDEC. Acerca del método utilizado para medir la pobreza en la Argentina. Buenos Aires: INDEC. 2003. [Acceso: 15 de febrero de 2018]. Disponible en: https: / / www.indec.gob. $\mathrm{ar} / \mathrm{ftp} /$ cuadros/sociedad/pobreza2.pdf.

17. Crino $M$, Barakat $T$, Trecena $H$, et al. Systematic review and comparison of classification frameworks describing the degree of food processing. Nutr Food Technol 2017;3(1):138.

18. MoubaracJC, Parra DC, Cannon G, et al. Food Classification Systems Based on Food Processing: Significance and Implications for Policies and Actions: A Systematic Literature Review and Assessment. Curr Obes Rep 2014; $3(2): 256-72$

19. Kelly B, Jacoby E. Public Health Nutrition special issue on ultra-processed foods. Public Health Nutr 2018; 21(1):1-4.

20. FAO. Guidelines on the collection of information on food processing through food consumption surveys. Roma: FAO; 2015. [Acceso: 18 de diciembre de 2017]. Disponible en: http: / / www.fao.org/3/a-i4690e.pdf.

21. Batal M, Johnson-Down L, Moubarac JC, et al. Quantifying associations of the dietary share of ultra-processed foods with overall diet quality in First Nations peoples in the Canadian provinces of British Columbia, Alberta, Manitoba and Ontario. Public Health Nutr 2017; 21:103-13.

22. MonteiroCA, Cannon G, MoubaracJC, etal. TheUNDecade of Nutrition, the NOVA food classification and the trouble with ultra-processing. Public Health Nutr 2017; 21(1):5-17.

23. Costa Louzada ML, Martins A, Canella D, et al. Ultraprocessed foods and the nutritional dietary profile in Brazil. Rev Saude Publica 2015; 49:38.

24. Monteiro CA, Moubarac JC, Levy RB, et al. Household availability of ultra-processed foods and obesity in nineteen European countries. Public Health Nutr 2018; 21(1):18-26.

25. Monteiro CA, Levy RB, Claro RM, et al. Increasing consumption of ultra-processed foods and likely impact on human health: evidence from Brazil. Public Health Nutr 2011; 14(1): 5-13.

26. Ministerio de Justicia y Derechos Humanos. Secretaría de Políticas, Regulación Institutosy Secretaría de Agricultura, Ganadería y Pesca. Código Alimentario Argentino. Resolución Conjunta 137/2010 y 941/2010 Modificación. Buenos Aires, 3 de diciembre de 2010. [Acceso: 18 de diciembre de 2017]. Disponibleen: http:/ / servicios.infoleg. gob.ar/infolegInternet/anexos/175000-179999/176669/ norma.htm.

27. Galante M, Konfino J, Ondarsuhu D, et al. Principales resultados de la tercera Encuesta Nacional de Factores de Riesgo de enfermedades no transmisibles en Argentina. Rev Argent Salud Pública 2015; 6(24):22-9.

28. Datum Internacional. PERCEPCIÓN Y REALIDAD. Un estudio sobre obesidad en América. Octubre de 2014. [Acceso: 15 de febrero de 2018]. Disponible en: http:/ / docplayer.es/15684192-Percepcion-y-realidad-un-estudiosobre-obesidad-en-america-octubre-2014.html.

29. Zapata ME. Patrón de consumo de bebidas en Argentina: resultados de los estudios Hidratar I y II. En: Carmuega E. (editor). Hidratación saludable en la infancia. Buenos Aires: CESNI, 2015.Págs.119-41.

30. Euromonitor International. Carbonates in Argentina. 2017. [Acceso: 18 de diciembre de 2017]. Disponible en: http:/ / www.euromonitor.com/carbonates-in-argentina/report.

31. Zapata ME, Rovirosa A, Carmuega E. Cambios en el patrón de consumo de alimentos y bebidas en Argentina, 19962013. Salud Colectiva 2016; 12(4):473-86.

32. Gibson R, Charrondiere R, Bell W. Measurement errors in dietary assessment using self-reported 24-hour recalls in low-income countries and strategies for their prevention. Adv Nutr 2017; 8(6):980-91.

33. Vandevijvere S, Monteiro C, Krebs-Smith SM, et al. Monitoring and benchmarking population diet quality globally: a step-wise approach. Obes Rev 2013; 14(Suppl 1):135-49.

34. Popkin BM, Adair LS, Ng SW. Now and then: The global nutrition transition: the pandemic of obesity in developing countries. Nutr Rev 2012; 70(1):3-21. 


\section{ANEXO}

TABLA 1. Número de casos encuestados según grupo etario y región

\begin{tabular}{lccc}
\hline Región & \multicolumn{1}{c}{$\begin{array}{c}\text { Grupo etario } \\
\text { Niños y niñas de } \mathbf{6} \text { a 23 meses }\end{array}$} & $\begin{array}{c}\text { Niños y niñas de 2 a 5 años } \\
\text { Mujeres de 10 a 49 años }\end{array}$ \\
\hline $\begin{array}{l}\text { AMBA } \\
\text { (CABA y partidos del conurbano) }\end{array}$ & 682 & 1012 & 854 \\
$\begin{array}{l}\text { Cuyo (Mendoza, San Juan y San Luis) } \\
\text { NEA (Chaco, Corrientes, Formosa y Misiones) }\end{array}$ & 1004 & 1230 & 1089 \\
$\begin{array}{l}\text { NOA (Catamarca, Jujuy, La Rioja, Salta, } \\
\text { Santiago del Estero y Tucumán) }\end{array}$ & 1042 & 1270 & 1169 \\
$\begin{array}{l}\text { Pampeana (resto de Buenos Aires, Córdoba, } \\
\text { Entre Ríos y Santa Fe) }\end{array}$ & 1203 & 1361 & 1259 \\
$\begin{array}{l}\text { Patagonia (Chubut, La Pampa, Neuquén, } \\
\text { Río Negro, Santa Cruz y Tierra del Fuego) }\end{array}$ & 966 & 1221 & 1047 \\
País & 1138 & 1270 & 1187 \\
\hline
\end{tabular}

Fuente: ENNyS 2005.

AMBA: Área Metropolitana de Buenos Aires; CABA: Ciudad Autónoma de Buenos Aires; NEA: Noreste Argentino; NOA: Noroeste Argentino.

TABla 2. Promedio de contenido nutricional de los alimentos consumidos según el grupo NOVA. Niños y niñas de 6 a 23 meses. Total del país. $N=6035$

\begin{tabular}{|c|c|c|c|c|c|}
\hline & Total & G1 & G2 & G3 & G4 \\
\hline Energía (kcal) & 1175,4 & 558,7 & 161,3 & 138,2 & 317,2 \\
\hline Proteínas (g) & 45,3 & 31,7 & 0,0 & 5,5 & 8,1 \\
\hline AG saturados ${ }^{1}$ & 13,2 & 15,9 & 8,9 & 11,6 & 9,6 \\
\hline AG monoinsaturados ${ }^{1}$ & 10,1 & 9,7 & 13,7 & 6,6 & 9,8 \\
\hline AG poliinsaturados ${ }^{1}$ & 5,9 & 2,3 & 26,4 & 1,3 & 3,3 \\
\hline Lípidos $(\mathrm{g})$ & 41,4 & 20,0 & 8,7 & 3,1 & 9,6 \\
\hline Hidratos disponibles (g) & 155,5 & 63,1 & 20,8 & 22,0 & 49,6 \\
\hline Fibra $(\mathrm{g})^{2}$ & 5,8 & 3,7 & 0,0 & 1,1 & 1,0 \\
\hline Sodio (mg) & 855,4 & 316,8 & 2,7 & 147,1 & 388,8 \\
\hline Calcio (mg) & 766,6 & 573,1 & 0,4 & 57,9 & 135,2 \\
\hline
\end{tabular}

G1: alimentos no procesados; G2: ingredientes; G3: alimentos procesados; G4: alimentos ultraprocesados.

AG: ácidos grasos; Kcal: kilocalorías; G: grupo; g: gramos; mg: miligramos.

${ }^{1}$ : Porcentaje sobre el total de la energía consumida.

2: Estimado solo para niños y niñas de 13 a 23 meses por no haber recomendaciones para niños y niñas menores.

TABla 3. Promedio de contenido nutricional de los alimentos consumidos según el grupo NOVA. Niños y niñas de 2 a 5 años. Total del país. $N=7364$

\begin{tabular}{|c|c|c|c|c|c|}
\hline & Total & G1 & G2 & G3 & G4 \\
\hline Energía (kcal) & 1683,5 & 646,1 & 234,7 & 262,3 & 540,4 \\
\hline Proteínas (g) & 59,9 & 39,3 & 0,0 & 9,5 & 11,0 \\
\hline AG saturados ${ }^{1}$ & 11,8 & 15,1 & 9,7 & 10,8 & 8,2 \\
\hline AG monoinsaturados ${ }^{1}$ & 10,3 & 10,3 & 13,9 & 6,9 & 9,3 \\
\hline AG poliinsaturados ${ }^{1}$ & 6,4 & 2,6 & 26,2 & 1,4 & 3,9 \\
\hline Lípidos (g) & 58,4 & 22,7 & 13,9 & 5,4 & 16,5 \\
\hline Hidratos disponibles (g) & 229,6 & 71,2 & 27,4 & 44,0 & 86,9 \\
\hline Fibra $(\mathrm{g})$ & 8,6 & 4,7 & 0,0 & 2,0 & 1,9 \\
\hline Sodio (mg) & 1152,0 & 329,7 & 6,1 & 266,3 & 550,0 \\
\hline Calcio (mg) & 761,0 & 540,3 & 0,7 & 81,1 & 138,9 \\
\hline
\end{tabular}

G1: alimentos no procesados; G2: ingredientes; G3: alimentos procesados; G4: alimentos ultraprocesados.

AG: ácidos grasos; Kcal: kilocalorías; G: grupo; g: gramos; mg: miligramos.

${ }^{1}$ : Porcentaje sobre el total de la energía consumida. 
II / Arch Argent Pediatr 2018;116(5):345-352 / Artículo especial

TABla 4. Promedio de contenido nutricional de los alimentos consumidos según el grupo NOVA. Mujeres de 10 a 49 años. Total del país. $N=6605$

\begin{tabular}{|c|c|c|c|c|c|}
\hline & Total & G1 & G2 & G3 & G4 \\
\hline Energía (kcal) & 2038,4 & 635,2 & 370,0 & 441,9 & 591,3 \\
\hline Proteínas (g) & 68,9 & 40,0 & 0,0 & 16,1 & 12,8 \\
\hline AG saturados ${ }^{1}$ & 10,0 & 10,7 & 8,8 & 10,7 & 8,7 \\
\hline AG monoinsaturados ${ }^{1}$ & 10,2 & 9,7 & 13,3 & 7,0 & 10,1 \\
\hline AG poliinsaturados ${ }^{1}$ & 6,7 & 2,7 & 23,0 & 1,4 & 4,6 \\
\hline Lípidos (g) & 67,9 & 17,4 & 18,7 & 9,7 & 22,1 \\
\hline Hidratos disponibles $(\mathrm{g})$ & 250,6 & 61,1 & 41,7 & 55,7 & 92,1 \\
\hline Fibra (g) & 13,4 & 7,2 & 0,0 & 3,3 & 2,9 \\
\hline Sodio (mg) & 1391,4 & 235,5 & 7,8 & 463,5 & 684,6 \\
\hline Calcio (mg) & 473,4 & 210,9 & 0,9 & 151,2 & 110,3 \\
\hline
\end{tabular}

G1: alimentos no procesados; G2: ingredientes; G3: alimentos procesados; G4: alimentos ultraprocesados. AG: ácidos grasos; Kcal: kilocalorías; G: grupo; g: gramos; mg: miligramos.

${ }^{1}$ : Porcentaje sobre el total de la energía consumida. 